This item was submitted to Loughborough's Research Repository by the author.

Items in Figshare are protected by copyright, with all rights reserved, unless otherwise indicated.

\title{
30-GHz high-frequency application of screen printed interconnects on an organic substrate
}

PLEASE CITE THE PUBLISHED VERSION

https://doi.org/10.1109/TCPMT.2017.2718023

PUBLISHER

(C) IEEE

VERSION

AM (Accepted Manuscript)

LICENCE

CC BY-NC-ND 4.0

\section{REPOSITORY RECORD}

Lim, Ying Ying, Yee Goh, Manuba Yoshida, Tung T. Bui, Masahiro Aoyagi, and Changqing Lui. 2019. "30-ghz High-frequency Application of Screen Printed Interconnects on an Organic Substrate". figshare.

https://hdl.handle.net/2134/25438. 


\section{$30 \mathrm{GHz}$ High Frequency Application of Screen Printed Interconnects on an Organic Substrate}

\begin{tabular}{|r|l|}
\hline Journal: & Transactions on Components, Packaging and Manufacturing Technology \\
\hline Manuscript ID & TCPMT-2016-300.R1 \\
\hline Manuscript topic: & ELECTRICAL PERFORMANCE OF INTEGRATED SYSTEMS \\
\hline Date Submitted by the Author: & n/a \\
\hline Complete List of Authors: & $\begin{array}{l}\text { Lim, Ying Ying; National Institute of Advanced Industrial Science and } \\
\text { Technology (AIST), Nanoelectronics Research Institute } \\
\text { Goh, Yee Mey; Loughborough University, Wolfson School of Mechanical \& } \\
\text { Manufacturing Engineering } \\
\text { Yoshida, Manabu; National Institute of Advanced Industrial Science and } \\
\text { Technology (AIST), Flexible Electronics Research Center (FLEC) } \\
\text { Bui, Tung; National Institute of AIST, Nanoelectronics Research Institute; } \\
\text { Vietnam National University, Electronics and Telecomunication } \\
\text { Aoyagi, Masahiro; National Institute of Advanced Industrial Science and } \\
\text { Technology (AIST), Nanoelectronics Research Institute } \\
\text { Liu, Changqing; Loughborough University, Wolfson School of Mech \& Man } \\
\text { Eng. }\end{array}$ \\
\hline \multirow{2}{*}{ Keywords: } & \begin{tabular}{l} 
screen printing, Transmission lines, RF performance \\
\hline
\end{tabular} \\
\hline
\end{tabular}




\title{
$30 \mathrm{GHz}$ High Frequency Application of Screen Printed Interconnects on an Organic Substrate
}

\author{
Ying Ying Lim, Yee Mey Goh, Manabu Yoshida, Tung Thanh Bui, \\ Masahiro Aoyagi, Senior Member, IEEE, Changqing Liu, Senior Member, IEEE
}

\begin{abstract}
Printed conductive traces on flexible substrates offer many potential applications in the area of wearable electronics, ranging from search and rescue operations to health and physiological monitoring. Literature abounds on the effect of sintering conditions on the DC electrical resistivity of printed traces, due to the applications considered which fall in the lower frequency domain (megahertz range). There is a growing interest to investigate Wireless Body Area Networks (WBANs) for wearable electronics operating in the higher frequencies, due to the advantages involved. At present there is little information available on the radio frequency (RF) performance of printed interconnects, and this work seeks to investigate the effect of the paste property on the DC conductivity and high frequency performance $(\leq 30 \mathrm{GHz})$ of interconnects. The results obtained suggest that paste levelling has a significant influence on the DC electrical performance. In addition, the DC conductivity values are possibly affected by the adhesion of the paste onto the particular substrate during the printing process, which was observed to have a significant effect on the quality and thicknesses of the traces printed. Lastly, the influence of the DC conductivity on the high frequency performance of interconnects is investigated, where the measured results are validated with simulation results.
\end{abstract}

Index Terms - DC conductivity, RF performance, screen printing, silver nanoparticle paste, transmission lines

\section{INTRODUCTION}

$T_{t}^{\mathrm{H}}$ HE emergence of the Internet of Things (IoT) is anticipated to become a reality in the future when sensors and internet connectivity would enable individual devices to communicate. In particular, the merging of wireless technology with consumer electronics has made wearable electronics increasingly pervasive in everyday life, with applications spanning from the health and physiological monitoring of the wearer (e.g. patients, athletes) to military operations.

Currently most Wireless Body Area Networks (WBANs) for wearable electronics operate in the IEEE 802.15.4 Zigbee standard [1], and there is a growing interest to investigate the performance of BAN in the millimeter wave band (e.g. $60-\mathrm{GHz}$

Y.Y. Lim, M. Yoshida and M. Aoyagi are with the National Institute of Advanced Industrial Science and Technology (AIST), Tsukuba, Ibaraki, 305-8560 Japan (e-mail: yingying.lim@aist.go.jp).

Y.M. Goh and C. Liu are with the Mechanical, Electrical \& Manufacturing Engineering, Loughborough University, Epinal Way, Loughborough, Leicestershire, LE11 3TU, UK (e-mail: c.liu@ lboro.ac.uk).

T.T. Bui was with the National Institute of Advanced Industrial Science and Technology (AIST) and is now with the University of Engineering and Technology, Vietnam National University, Hanoi, Vietnam. band) due to the potential advantages. One advantage lies in the higher data rates which can be obtained, which translates to reduced transmission times. Another advantage lies in the increased security and lower interference with adjacent WBANs, due to the stronger attenuation of these signals in the atmosphere.

The conductive traces for wearable electronics can be realized using various techniques, among which are screen printing and inkjet printing. Yet the electrical performances of printed traces in the higher frequency range (gigahertz range) have been subjected to little investigation to date [2]. Rather, work abounds on the effect of sintering conditions on the DC electrical resistivity of printed traces [2]-[7], due to the applications considered which are in the lower frequency domain (e.g. RFID tags operating at megahertz frequencies, solar cells). While Kim et al. [2] reported the RF characterization of inkjet-printed transmission lines up to 30 $\mathrm{GHz}$ on silicon, little information was available on whether the $\mathrm{RF}$ results were realistic or within a reasonable benchmark. In addition, Salmeron et al. [8] used a 2.5D electromagnetic simulator to evaluate the electrical performance of transmission lines against the fabricated structures, but only up to a frequency of $1.2 \mathrm{GHz}$. In addition the simulator is not able to take into account conductor loss effects arising from the finite thickness and conductivity of the actual printed 3D structure.

Due to a lack of research, there is little information available on the RF performance of printed interconnects. Printed interconnects in this context refer to those obtained by screen printing, but the methodology proposed would be applicable for other types of printed interconnects (e.g. inkjet printed traces).

The main objective of this work is to investigate the influence of the paste property on the DC conductivity of printed interconnects across a substrate, and the corresponding RF performance up to $30 \mathrm{GHz}$. In this case, the focus is not on modifying the paste formulation or properties, but on understanding how specific paste properties affect the electrical performance of printed interconnects. The RF measurements are validated with simulation models which take into account non-ideal transmission line characteristics (typically neglected during the design and modelling process), so as to accurately predict the RF response. To date, there exists little literature which reports on the DC conductivity variation of printed samples across a substrate, since most researchers focus on measuring samples for repeatability [2], or samples of a different variety [9], [10]. 


\section{MATERIALS}

\section{1) Substrate}

The substrate used in this work (RO3006) is a ceramic-filled PTFE laminate typically used for high frequency applications [11], with dielectric properties well characterized up to $40 \mathrm{GHz}$ (Dielectric constant $=6.15 \pm 0.15$, loss tangent $=0.002$ ) $[11]$. The laminate is supplied in a $12 \times 9$ inch sheet with a laminate thickness of $1.28 \mathrm{~mm}$ and $17 \mu \mathrm{m}$ electrodeposited copper on both sides. In order to prepare a single-sided laminate, the copper on one side of the laminate was chemically removed using the standard printed circuit board (PCB) process lamination, UV exposure, development, etching and stripping. The single-sided laminate is used for the trace deposition using screen printing. The reference substrate used in this paper is borosilicate glass (Borofloat ${ }^{\circledR} 33$ ), which has $1 \mathrm{~mm}$ thickness (Dielectric constant $=4.6$, loss tangent $=37 \times 10^{-4}$ at $1 \mathrm{MHz}$ ).

\section{2) Silver Pastes}

Two nanoparticle-based silver pastes were used in this work, which were selected based on their suitability for fine line printing. The first paste is Rexalpha (RA FS 088, Toyo Co. Ltd), which has a viscosity of 85 Pa.s and a silver content of $65-70 \mathrm{wt} \%$. From the datasheet, the resistivity values are $8 \mathrm{x}$ $10^{-5} \Omega$-cm $\left(130{ }^{\circ} \mathrm{C}, 30\right.$ minutes $)$ and $10 \times 10^{-5} \Omega-\mathrm{cm}\left(100{ }^{\circ} \mathrm{C}\right.$, 30 minutes). The second paste is a research sample (CA-T31, Daiken chemical Co. Ltd) with a higher viscosity of $229 \mathrm{~Pa} . \mathrm{s}$ and a silver content of $83.2 \mathrm{wt} \%$. The corresponding resistivity is $1.4 \times 10^{-5} \Omega-\mathrm{cm}\left(160{ }^{\circ} \mathrm{C}, 30\right.$ minutes $)$.

\section{METHODOLOGY}

The methodology used in this paper is outlined in Fig. 1, with the aim of designing $50 \Omega$ coplanar waveguide (CPW) transmission line structures. This necessitates a trial test to be performed to determine the paste spread for the silver paste and substrate combination used. The test structures for this trial include $50 \Omega \mathrm{CPW}$ structures (designed) with zero spread. In addition other CPW structures were considered, with the dimensions compensated to consider paste spread ranging from $25 \mu \mathrm{m}$ to $150 \mu \mathrm{m}$. The assumption made is that the paste spreads equally in the $\mathrm{x}$-direction for both the signal and ground traces. The details of the compensated dimensions for a $250 \mu \mathrm{m}$ pitch structure are shown in Table 1 . The CPW structures are designed with $250 \mu \mathrm{m}$ and $500 \mu \mathrm{m}$ pitch to allow for RF measurements using GSG probes. After the paste spread has been determined, the final $50 \Omega$ structures can then be designed and realized via screen printing.

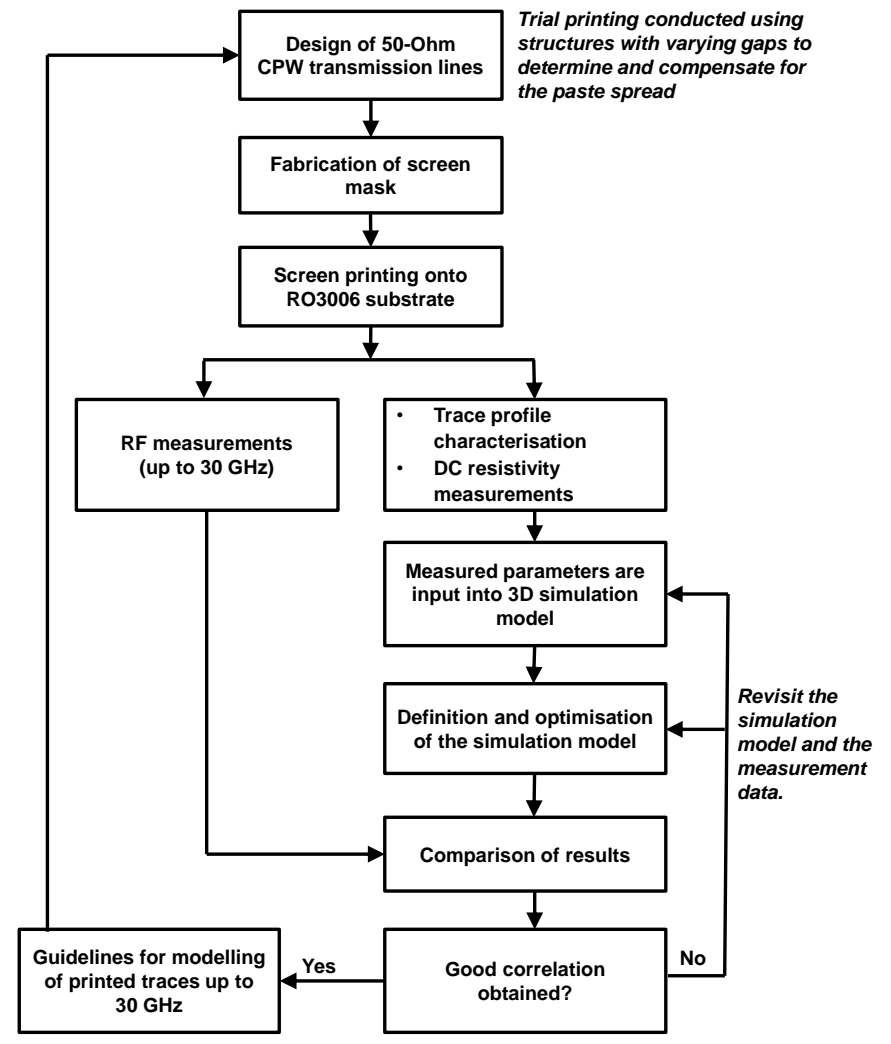

Fig. 1. A flowchart that outlines the approach used in this work.

TABLE I

SCREEN DESIGNS WHICH INCLUDE $50 \Omega$ CPW STRUCTURES WITH PASTE COMPENSATION

\begin{tabular}{lccccc}
\hline \hline & \multicolumn{5}{c}{ Width/spacing of CPW structure } \\
\cline { 2 - 6 } & $\begin{array}{l}0 \mu \mathrm{m} \\
\text { spread }\end{array}$ & $\begin{array}{l}25 \mu \mathrm{m} \\
\text { spread }\end{array}$ & $\begin{array}{r}50 \mu \mathrm{m} \\
\text { spread }\end{array}$ & $\begin{array}{r}100 \mu \mathrm{m} \\
\text { spread }\end{array}$ & $\begin{array}{c}150 \mu \mathrm{m} \\
\text { spread }\end{array}$ \\
\hline \hline $\begin{array}{l}\text { Screen design } \\
\begin{array}{l}\text { Printed } \\
\text { (Predicted) }\end{array}\end{array}$ & $150 / 45$ & $150 / 75$ & $150 / 100$ & $150 / 170$ & $150 / 230$ \\
\hline \hline
\end{tabular}

A number of techniques were used to characterize the samples in order to extract the trace profile and evaluate the electrical performance (DC and RF). These include four point probe measurements to obtain the DC resistivity of the printed traces. The trace profiles (e.g. signal width, signal-ground spacing) were characterized using a non-contact measurement technique. The data from these characterizations were then used to construct a simulation model in CST [9], which is a 3D electromagnetic simulator. After validating the simulation model and optimizing it (in terms of meshing), the simulation results are correlated with the RF measurements. A good correlation would then provide guidelines for the modelling of printed traces up to $30 \mathrm{GHz}$. If a poor correlation is obtained, the measured data used for the simulation model would be reconsidered. In addition, the assumptions made for the simulation model would be re-visited. 


\section{TEST STRUCTURES}

An overview of the screen mask is shown in Fig. 2(a). The screen mask is divided into four regions, where the test structures are repeated in each region. This is to determine the repeatability of the printed test structures across the four regions (i.e. across different regions of the substrate).

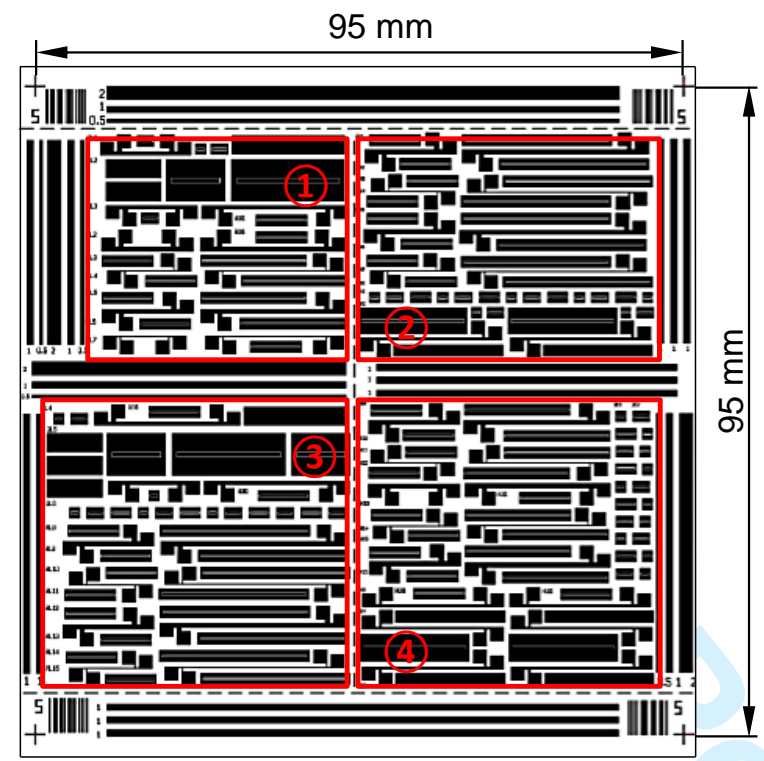

(a)

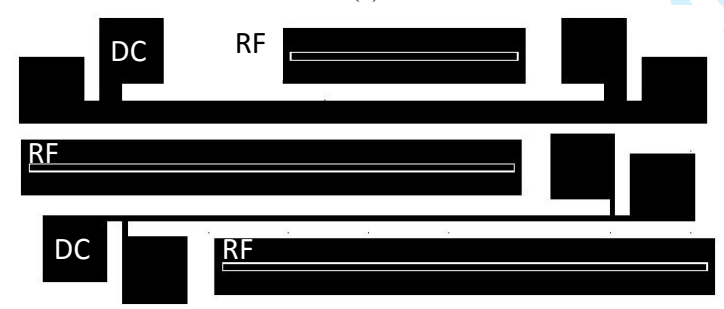

(b)

Fig. 2. Diagram showing (a) an overview of the screen mask designs indicating the 4 regions. (b) Magnified view of a section of the layout showing four point probe test structures adjacent to the CPW transmission lines. The four point probe structures are labelled with "DC" while the CPW transmission lines are labelled with "RF".

With reference to Fig. 2, each region comprises of the following test structures:

\section{1) $50 \Omega C P W$ transmission lines}

The transmission lines are designed for RO3006 and glass substrates. The linewidth/spacing (w/s) of the CPW dimensions for RO3006 is $150 / 75 \mu \mathrm{m}$. The CPW line lengths are $7 \mathrm{~mm}$ and $15 \mathrm{~mm}$.

\section{2) Four point probe test structures}

These are positioned adjacent to the CPW transmission lines (shown in Fig. 2(b)), such that an in-situ measurement of the DC resistivity could be made for each CPW line for the simulation correlation. The rationale is that using an average conductivity value for the simulation correlation may not suffice, thus the need for four point probe structures to be placed beside the measured CPW lines for in-situ measurements. The widths of the test structures correspond to the signal (w) and ground (g) dimensions of the CPW lines, and there are 4 samples per width located in each region.

\section{V.SIMULATION MODEL}

The CPW structure is simulated using a time domain (TD) solver in CST which is suitable for the evaluation of electrically large structures. The solver uses hexahedral meshing to simulate the structures. The accuracy set for the simulation to terminate is $-40 \mathrm{~dB}$. Due to the symmetry of the reflection and transmission behavior of the signals, the S-parameter symmetry option $\left(S_{11}=S_{22}\right)$ is utilized in the simulation. Through this setting, the simulation time can be reduced as the solver considers only the essential simulation runs.

Due to the small thickness of the printed silver traces (8-9 $\mu \mathrm{m})$, the calculated skin depth was within the same order of magnitude of the thicknesses at $30 \mathrm{GHz}$. This necessitated dense meshing within the trace thickness in order to capture the transmission line loss arising from the skin effect. In addition, the meshing at the trace edges was refined to capture the current crowding effect in those areas. The 3D model was validated by comparing the $\mathrm{S}$-parameter results with those obtained from a frequency domain (FD) solver available in the same software. The FD solver uses a different meshing (tetrahedral meshing) to simulate a structure and is more typically used for electrically small structures. From Fig. 3, a good match in the S-parameter

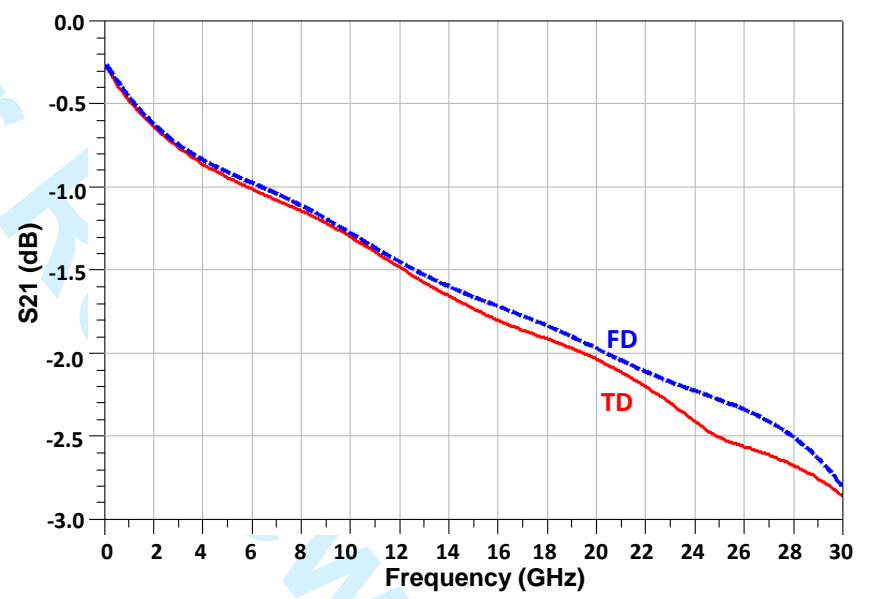

(a)

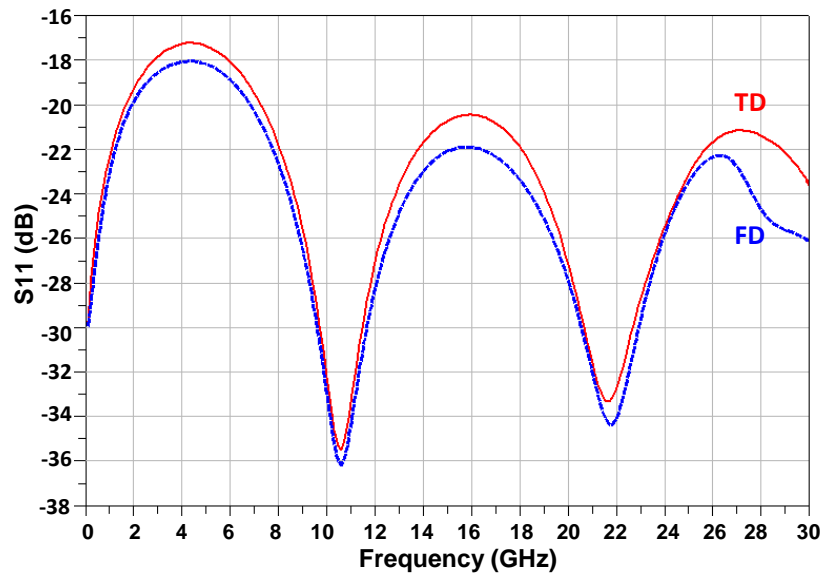

(b)

Fig. 3. Comparison of S-parameters obtained from TD and FD solvers in terms of the (a) S21 (b) S11. 
results is obtained, for a $7 \mathrm{~mm} \mathrm{CPW}$ structure on RO3006. The variation observed between the two set of results could be attributed to numerical error and the material characterization in the solvers, as the material properties are considered differently between the FD and TD models.

\section{EXPERIMENTAL DETAILS}

\section{1) Screen Printing}

A manual screen printer (TOPRO ZT320) was used for the screen printing, which had a suction function to hold the substrate in place on the metal chuck. In addition, tape was used to fix the 4 sides of the RO3006 substrate to the chuck, as a slight warpage was observed for the PTFE-based substrate. A screen size of $320 \times 320 \mathrm{~mm}$ was used, with a mesh count of 640 (i.e. 640 threads/inch), an emulsion thickness of $7 \mu \mathrm{m}$ and an open area of $39 \%$. A mesh tilt of $22.5^{\circ}$ was used for the screen. The test structures shown in Fig. 2 were fabricated in a smaller area of $100 \times 100 \mathrm{~mm}$. This is due to the limitation on the total design area possible (with respect to the screen size) in order to maintain the dimensional accuracy of the printed structures. Specifically the usable area was set at $100 \times 100 \mathrm{~mm}$ in accordance with the guideline of limiting the ratio between the design area and frame size to be within 1/9.

Prior to printing, the sample surfaces were cleaned with acetone and dried using nitrogen gas. The squeegee durometer (i.e. physical hardness) used for printing is $80-85$, and the print speed is around $200 \mathrm{~mm} / \mathrm{s}$. The off-contact distance between the mesh and substrate was set at $1.5 \mathrm{~mm}$. The samples were thermally cured at $130{ }^{\circ} \mathrm{C}$ for 1.5 hours after printing.

\section{2) Characterizing the CPW Transmission Line Profile}

To obtain a good correlation between simulation and measurement results, an accurate evaluation of the transmission line dimensions for use in the simulation model is critical. Fig. 4 is a schematic showing the evaluation of the transmission line dimensions, where the transmission line is divided into $\mathrm{N}$ sections.

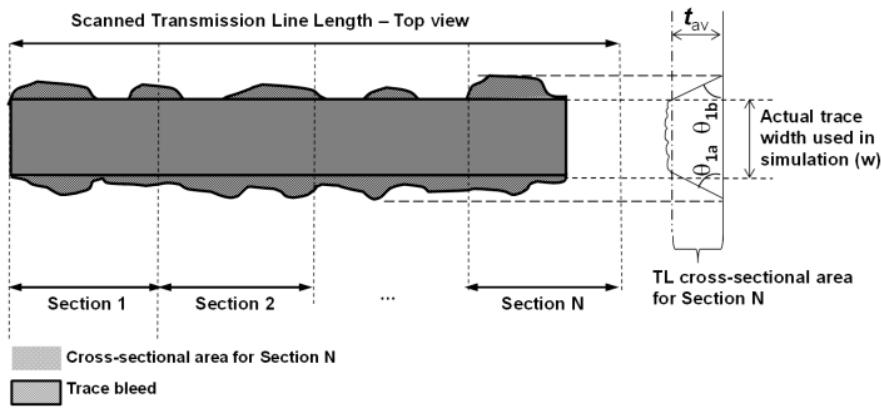

Fig. 4. Schematic showing the evaluation of the transmission line parameters measured using a non-contact technique.

The entire transmission line profile is scanned using the 3D non-contact measurement system (LEXT OLS-4100, Olympus) using a 20 objective lens which had a field of view of $80 \mu \mathrm{m}$. In particular, a differential interference contrast (DIC) - laser imaging option was used for the scan. This option provides a more accurate view of uneven surfaces (in range of a few nanometers to a few micrometers), and is used for the RO3006 substrate which has non-negligible surface roughness. During measurements the brightness from the lens is automatically adjusted at each acquisition position.

During the post-processing, levelling was performed for the scanned transmission line images. For glass substrates, an "inclination profile" option was used. However a manual correction was performed for the RO3006 substrate which displayed warpage after thermal curing, as RO3006 is a soft substrate. In this case the trace profile was corrected through the selection of 3 points on the image so as to specify the inclination plane for which to correct.

A profile width function was then used to extract the parameters of individual sections of the transmission line (Fig. 4). These parameters include the line width, height, cross-sectional area and trace edge angles. Each section was defined to be around 10-20\% of the total transmission line length, from which the averaged values of the respective parameters were obtained. The number of sections $(\mathrm{N})$ considered for the RO3006 substrate was increased because some of the areas of the substrate are warped. As a result, the regions which can be considered level are smaller. In particular, the regions with trace bleeding were not considered in the extraction of the trace width as the corresponding thicknesses were observed to be negligible. From literature, Aoki et al. [12] observed that the bleeding regions are not connected and as such may not contribute to the current flow.

Using the methodology described above, the profiles for a CPW structure as shown in Fig. 5 were characterized. This includes the signal width $(w)$, the signal-ground spacings $\left(g_{1}\right.$, $\left.g_{2}\right)$, the conductor edge angles $\left(\theta_{1 \mathrm{a}}\right.$ to $\left.\theta_{3 \mathrm{~b}}\right)$, and the conductor thicknesses $(t)$. The average conductor thickness for the signal and ground traces was used for $t$ in the simulation correlation.

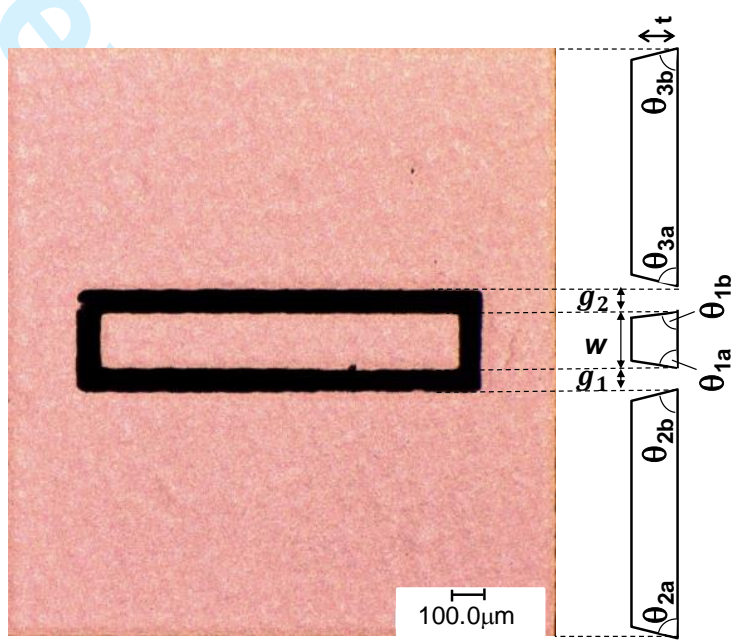

Fig. 5. A typical CPW structure with notations indicating the parameters extracted from the trace profile.

The angles $\theta_{1 \mathrm{a}}, \theta_{1 \mathrm{~b}}$ used in the simulation correlation were compensated by $5^{\circ}$ since the trace bleed regions on the trace periphery were excluded in the extraction of the trace width. The trace dimensions were extracted with an accuracy of around $\pm 10 \mu \mathrm{m}$. 


\section{3) Characterizing the DC Test Structure}

The same x20 objective lens with DIC-laser option was used to characterize the DC test structures. The measurements are made for the two ends of the test structures and the results averaged, with the assumption that the dimensions of the test structures are consistent. For each scanned region of the test structure, the average cross-sectional area can be calculated using a post processing function. The average trace thickness is determined manually using measurement lines in the software.

\section{4) DC Measurements}

The four point probe measurements were performed using a semiconductor parameter analyzer (Agilent 4156C) connected to a DC probe station. The input current range was set from -1 $\mathrm{mA}$ to $1 \mathrm{~mA}$ in steps of $10 \mu \mathrm{A}$ (i.e. 201 data points) and the corresponding voltage obtained from the four point probe structures. Each structure was measured twice, and the average value of 2 measurements was used to calculate the DC resistivity value. For each region, four samples of $150 \mu \mathrm{m}$ trace widths and four samples of $690 \mu \mathrm{m}$ trace widths were measured.

\section{5) $R F$ Measurements}

The RF measurement was performed over a frequency range from $0.1-30.1 \mathrm{GHz}$ with 201 measurement points. Microwave coplanar probes with $250 \mu \mathrm{m}$ pitch (40A-GSG-250-DP, GGB Industries Inc.) were used for the measurements. An intermediate frequency (IF) bandwidth of $100 \mathrm{~Hz}$ was used along with an averaging factor of 64 . Prior to measurements, a LRRM calibration was used and the magnitude of the $S_{11}$ parameter for a $600 \mu \mathrm{m}$ coplanar line standard on the calibration substrate was verified to be $\leq-40 \mathrm{~dB}$ at $30.1 \mathrm{GHz}$. The test structure was measured twice for verification.

\section{RESULTS AND DISCUSSION}

\section{1) Paste Characteristics on DC Conductivity}

Fig. 6 shows the results obtained for DC test structures on RO3006 with widths of $150 \mu \mathrm{m}$ and $690 \mu \mathrm{m}$. The widths correspond to the signal and ground dimensions of a CPW line and the trace thicknesses range from 8-9 $\mu \mathrm{m}$ on RO3006 substrate.

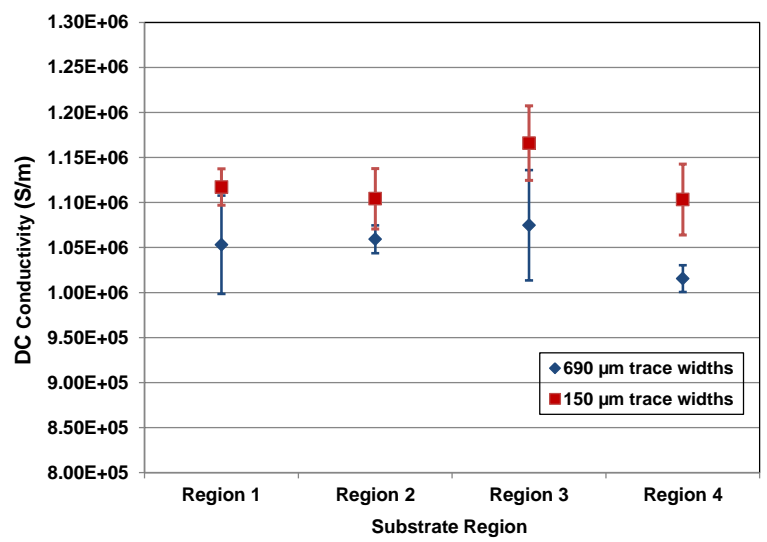

Fig. 6. Standard deviation plot of the extracted DC conductivity in the different regions of the RO3006 panel.

From the results it can be observed that the average DC conductivity values after 1.5 hours of thermal cure were close to that provided by the manufacturer after 30 minutes of thermal curing $\left(1.25 \times 10^{6} \mathrm{~S} / \mathrm{m}\right)$, which suggests that the DC conductivity may have reached saturation point due to paste fillers present in the formulation. This implies that obtaining lower DC resistivity values may only be possible through the use of a different paste formulation. However, the values were noted to vary across the 4 regions of the panel in Fig. 6.

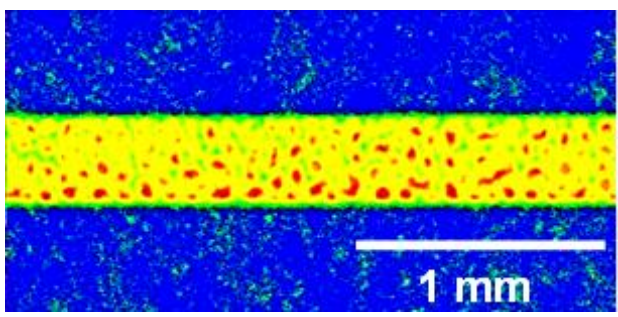

(a)

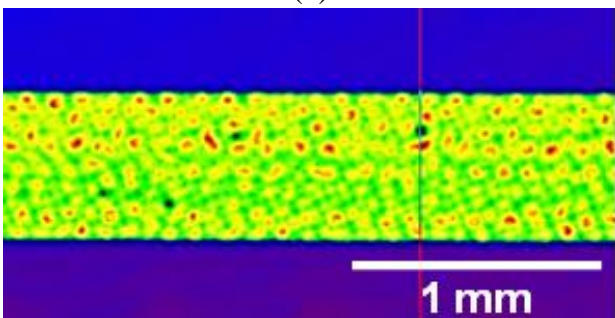

(b)

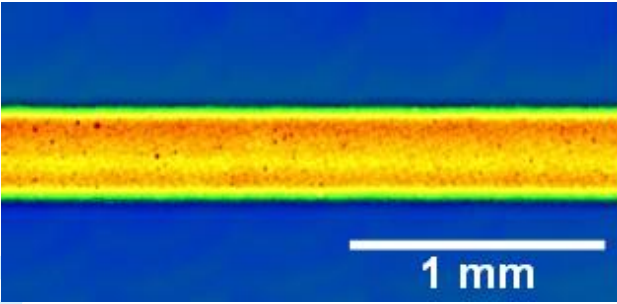

(c)

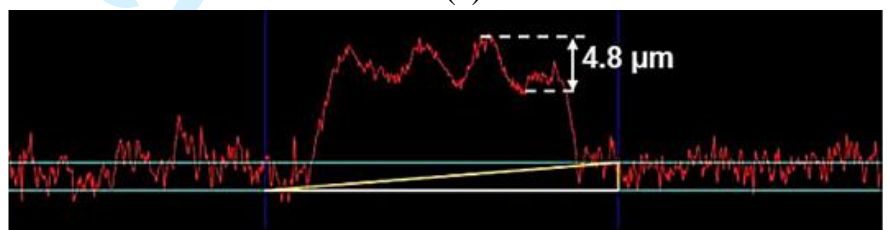

(d)

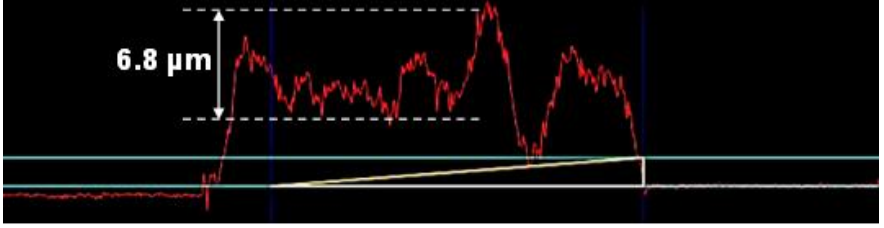

(e)

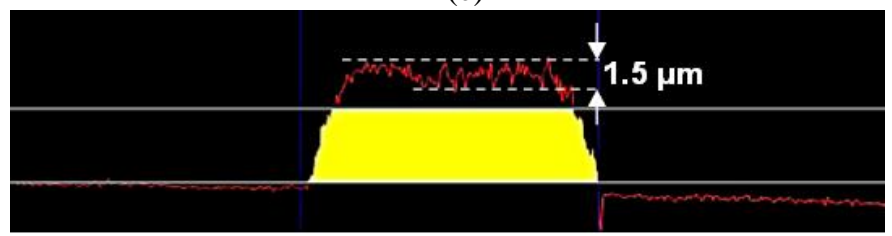

(f)

Fig. 7. Diagram showing trace profiles of DC test structures (a)Top view of trace on RO3006 using paste 1 ; (b) Top view of trace on glass using paste 1; (c) Top view of trace on glass using paste 2; (d) Corresponding cross-section of trace in (a); (e) Corresponding cross-section of trace in (b); (f) Corresponding cross-section of trace in (c). 
For the $150 \mu \mathrm{m}$ trace widths, the DC conductivity ranges from $1.10-1.17 \times 10^{6} \mathrm{~S} / \mathrm{m}$ while for the $690 \mu \mathrm{m}$ trace widths the DC conductivity is in the range of $1.02-1.07 \times 10^{6} \mathrm{~S} / \mathrm{m}$. The variation of the DC conductivity values may be attributed to the assumptions made during the extraction of the values using the four point probe measurement method. This technique assumes for a uniform material (i.e. uniform thickness) over the measurement area. In contrast the printed traces on RO3006 were observed to yield non-uniform thicknesses as shown in Fig. 7.

In Fig. 7(a)-(c), the red dots/regions observed represent the maximum trace thicknesses. From Fig. 7(a) and Fig. 7(b), the traces printed onto RO3006 and glass substrates (using paste 1) were observed to yield non-uniform thicknesses. This is also reflected in the corresponding cross-section profiles in Fig. 7(d) and Fig. 7(e), where a height variation of 4.8-6.3 $\mu \mathrm{m}$ was observed for printed traces using paste 1 . In contrast, a smaller thickness variation of $1.5 \mu \mathrm{m}$ was observed in Fig. 7(f) for printed traces on glass using paste 2 . This particular trace was also observed to have a more uniform thickness from the top profile captured in Fig. 7(c). Such a phenomenon could be mainly attributed to the paste levelling [13]-[14] after screen printing, which results from the screen mask and paste interaction. Itoh et al. [15] and Buzby et al. [14] highlighted that the paste transfer from the screen mask to the substrate is non-ideal, because the paste adhesion is also affected by the wire meshes and the emulsion side walls apart from the substrate surface. Buzby et al. [14] showed that the emulsion side walls are not perfectly smooth, which could have resulted in some paste adhesion to the side walls during printing. In addition, there would be some residual paste on the wire meshes during screen printing.

During the printing process, the paste formulation [13]-[14] influences the extent of adhesion of the paste onto the mesh and substrate, which in turn affects the uniformity profile of the printed trace. Phair et al. [13] suggested that increased binder content in the paste formulation would increase the adhesion property of the paste onto the mesh, which results in an increased surface roughness of the printed trace. This is consistent with the results obtained in Fig. 7, and information from the paste datasheets indicate that paste 1 has a higher binder content of 8-10 wt \%, compared to a binder content of $5-7 \%$ for paste 2 . From literature [13], the uniformity profile of the trace resulting from the screen mask-paste interaction has been reported to affect the mechanical and electrochemical characteristics [13] of the trace. The results obtained in this section suggest that this may also have a significant influence on the DC electrical performance.

Another interesting phenomenon was observed in comparing the results between RO3006 and borosilicate glass. From Fig. 8 , the DC conductivity range of the traces on RO3006 was noted to be consistently higher compared to those on borosilicate glass. In particular, the extracted DC conductivity of the traces on RO3006 is around $1.1 \times 10^{6} \mathrm{~S} / \mathrm{m}$, compared to a range of $5.4 \times 10^{5}-8.0 \times 10^{5} \mathrm{~S} / \mathrm{m}$ on borosilicate glass. This variation is by a factor of 1.4-2 times. Further investigation using optical microscopy (Fig. 9) reveals the presence of

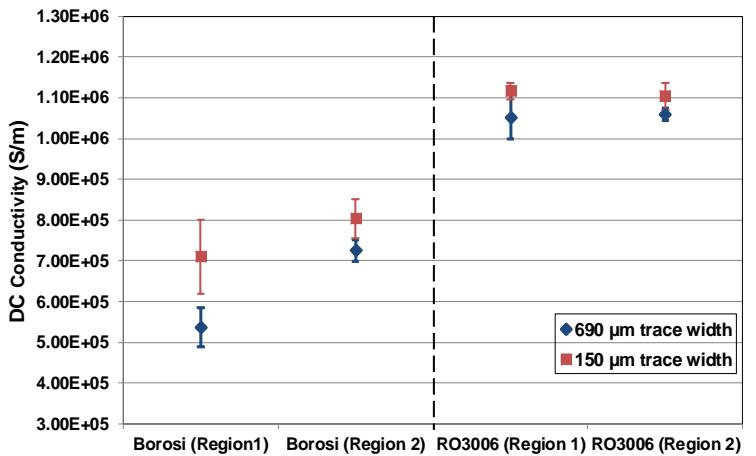

Fig. 8. DC conductivity of traces printed on borosilicate glass and RO3006.

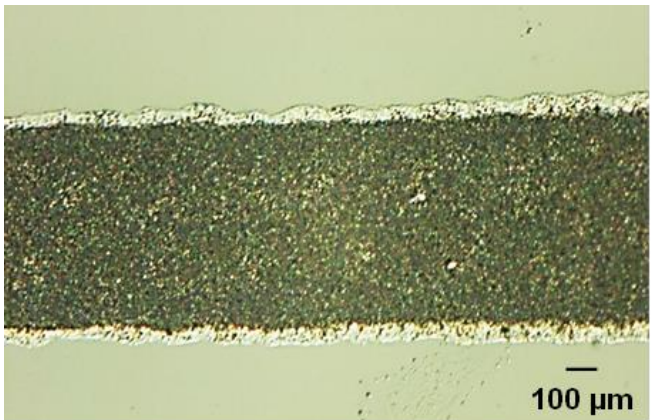

(a)

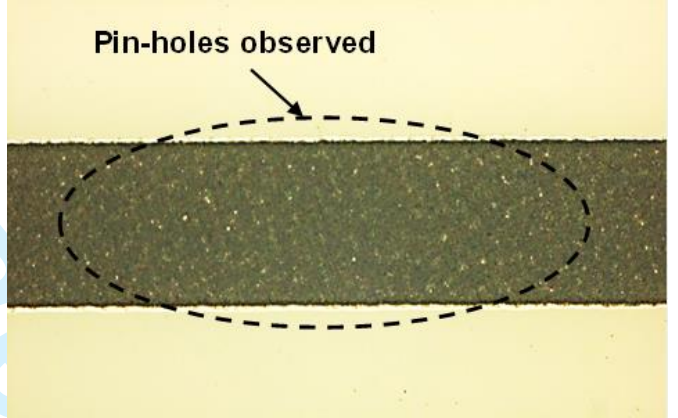

(b)

Fig. 9 Optical microscope images of silver traces after thermal curing showing (a) non-uniform thickness; (b) the presence of pin-holes.

pin-holes and mesh marks on the traces printed on borosilicate glass, with corresponding thicknesses of around 6-7 $\mu \mathrm{m}$. In particular, the presence of pin-holes would affect the current flow and the measured DC resistances, which are used to calculate the DC conductivity values.

It was also observed that while the trace thicknesses on borosilicate glass are within the recommended optimal trace thickness for paste $1(6-8 \mu \mathrm{m})$, the values fall in the lower range. From Equation (1):

$$
t_{f}=\left(\left(t_{\text {mesh }} x r_{\text {open }}\right)+t_{\text {emulsion }}\right) x r_{\text {wet-dry }}
$$

where $t_{f}$ represents the final trace thickness (after curing), $t_{\text {mesh }}$ is the mesh thickness, $r_{\text {open }}$ is the opening ratio, $t_{\text {emulsion }}$ is the emulsion thickness and $r_{w e t-d r y}$ is the wet-to-dry ratio. In this work, $t_{\text {mesh }}$ is $21 \mu \mathrm{m}$ and $r_{w e t-d r y}$ is 0.70 for paste 1 . Along with the paste parameters mentioned in Section $\mathrm{V}$, the calculated $t_{f}$ from (1) is $10.6 \mu \mathrm{m}$. However this value serves only as an estimate as (1) is based on the assumption that the paste is completely transferred from the 
screen mask to the substrate. Compared to the thickness range of 8-9 $\mu \mathrm{m}$ on RO3006, the results on borosilicate glass suggest that the final trace thickness is also affected by the adhesion of the paste onto the substrate, where a substrate with a higher surface roughness possibly results in a stronger adhesion force. Joo [16] suggested that the adhesion force would be reduced for substrate roughness which is an order of magnitude smaller than the particle diameter, since the particle-substrate contact area is smaller. In contrast, the adhesion force is expected to increase for substrate roughness which is comparable to the paste particle dimensions, due to an increased contact area between the paste particles and the substrate surface [16]. In this case the surface roughness on RO3006 is around $1 \mu \mathrm{m}$ [17], and the roughness values on borosilicate glass are below $3 \mathrm{~nm}$ [18]. From the FIB-SEM images in Fig. 10, the silver paste particle dimensions were observed in the order of hundreds of nanometers.

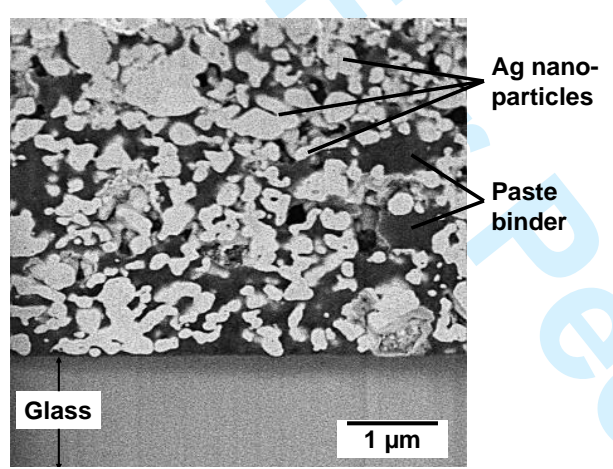

(a)

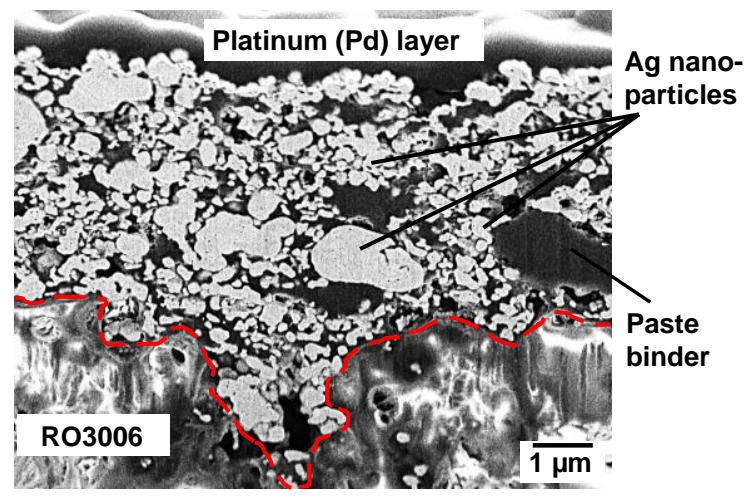

(b)

Fig. 10. FIB-SEM images of silver traces after thermal curing (a) on borosilicate glass; (b) on RO3006 substrate.

The relationship between the paste adhesion and the substrate roughness is also suggested by the trace width variation results obtained in Fig. 11, for designed trace widths of $150 \mu \mathrm{m}$ and $690 \mu \mathrm{m}$. While the traces deposited on RO3006 have higher thicknesses $(8-9 \mu \mathrm{m})$ compared to borosilicate glass $(6-7 \mu \mathrm{m})$, the corresponding trace spread is also consistently wider across the substrate. This suggests that the paste volume deposited on RO3006 is higher compared to borosilicate glass due to a stronger adhesion of the paste on RO3006.

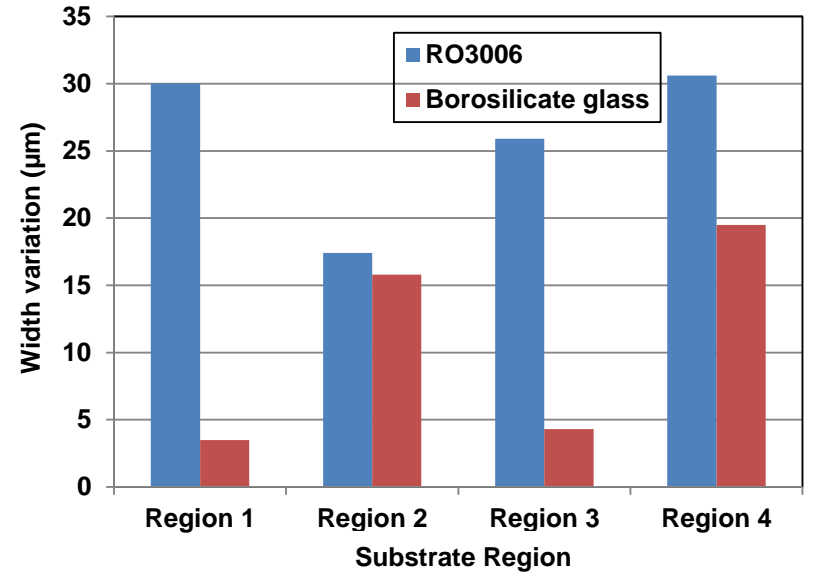

Fig. 11. Trace width variation across the different substrate regions for designed trace widths of $150 \mu \mathrm{m}$ and $690 \mu \mathrm{m}$.

Fig. 12 provides a summary of how the screen mask-paste interaction affects the printed trace profile.

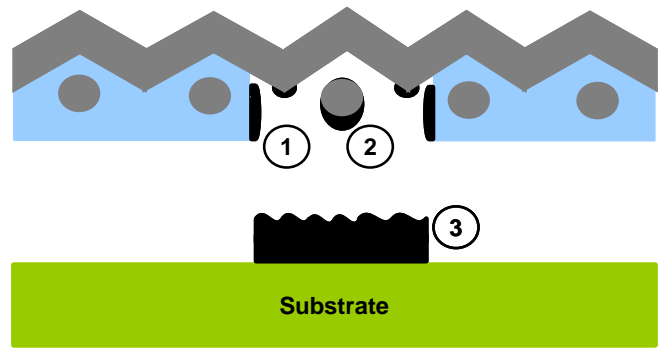

(1) Residual paste on emulsion side walls (not perfectly smooth).

(2) Residual paste on wire meshes.

(3) Adhesion of paste onto a particular substrate determines the quality of residual paste.

Fig. 12. Summary of factors influencing the paste-substrate interaction in the screen printing process.

Overall, the results necessitate the selection of a suitable paste which yields uniform trace thicknesses, to improve the consistency of the extracted DC conductivity values. This paste selection procedure is recommended before the actual screen printing of the test structures. The results also suggest that due to a variation in the DC conductivity values across the substrate, it is important to obtain accurate DC conductivity values for a

good simulation correlation with measurements for the printed transmission lines. It may be due to the conductivity and the presence of pin-holes in the screen printed traces that several researchers are focusing on applications at low frequencies such as RFID [8]-[9]. However one aim of this paper is to predict the performance of screen printed traces in the high frequency region ( $\mathrm{GHz}$ range), in the presence of pin-holes.

\section{2) Effect of Trace Edge on RF Performance}

The result correlation for a $2 \mathrm{~mm} \mathrm{CPW}$ line on RO3006 is shown in Fig. 13(a) and Fig. 13(b). The measured dimensions used in the simulation model are listed in Table 2, along with a measured DC conductivity value of $1.1 \times 10^{6} \mathrm{~S} / \mathrm{m}$. 


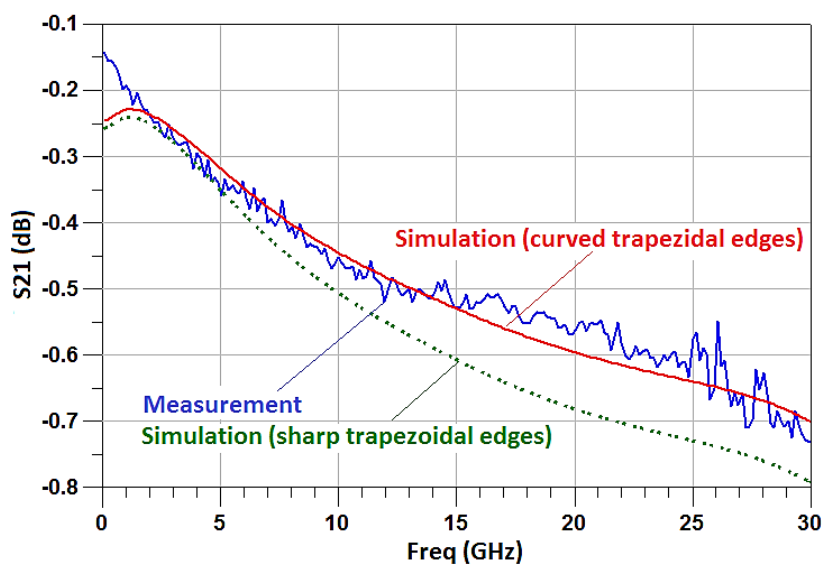

(a)

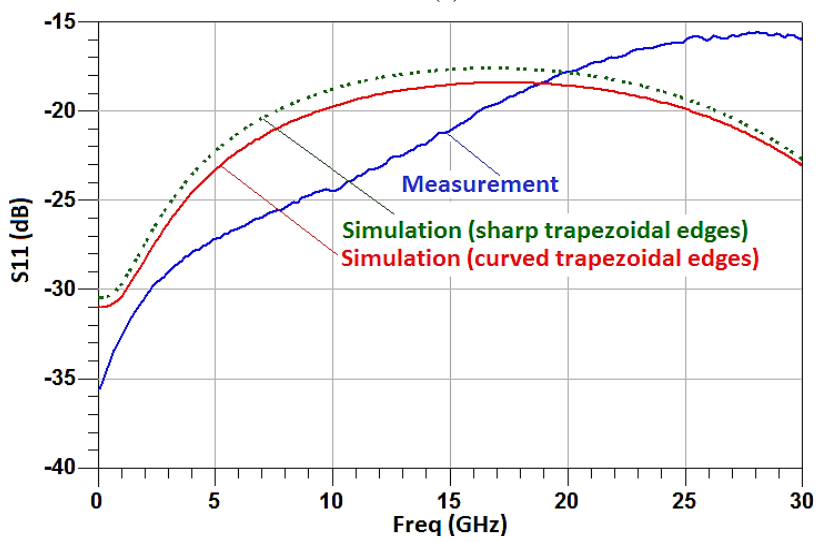

(b)

Fig. 13. Correlation between simulation and measurement results for a $2 \mathrm{~mm}$ CPW transmission line (a) Insertion loss; (b) Return loss.

TABLE II

TRACE PARAMETERS USED IN THE SIMULATION MODEL, WITH REFERENCE TO FIG. 5

\begin{tabular}{ccccc}
\hline \hline $\begin{array}{c}w \\
(\mu \mathrm{m})\end{array}$ & $\begin{array}{c}g_{1}, g_{2} \\
(\mu \mathrm{m})\end{array}$ & $\begin{array}{c}\mathrm{t} \\
(\mu \mathrm{m})\end{array}$ & $\begin{array}{c}\theta_{1 a}, \theta_{1 b} \\
\left({ }^{\circ}\right)\end{array}$ & $\begin{array}{c}\theta_{3 a}, \theta_{3 b} \\
\left({ }^{\circ}\right)\end{array}$ \\
\hline \hline 160 & 57,55 & 8.5 & $16.9,17.7$ & $17.9,13.1$ \\
\hline \hline
\end{tabular}

From Fig. 13, a good correlation can be observed between the simulation and measurement results up to $30 \mathrm{GHz}$. A discrepancy was observed at low frequency near DC in Fig. 13(a), which may be due to the desired accuracy not being attained in the low frequency region. In this case the maximum simulation time could be increased in the solver. From Fig. 13(b), the discrepancy between measurement and simulation results could be due to the modelling of the return path loop inductance. In the simulation model, waveguide ports were defined for excitation, where the ports were defined at both ends of the CPW transmission line. In this case the return path loop inductance of the test structure may not have been fully accounted for as a small segment of the CPW ground ring could not be modelled using the waveguide port setup. However a similar trend could be observed between the simulation and measurement results in Fig. 13(b), and the values of the return loss $\left(S_{11}\right)$ were observed to be $<-15 \mathrm{~dB}$, which suggests that the variation between simulation and measurement results can be considered negligible.

In particular, the effect of the trace edges was investigated for the $2 \mathrm{~mm}$ transmission line in the result correlation. When sharp trapezoidal edges were used to model the printed traces in the simulation model, the losses $\left(S_{21}\right)$ were found to be overestimated by about $14 \%$ at $30 \mathrm{GHz}$ as shown in Fig. 13(a), with the effect on the return loss being negligible (shown in Fig. 13(b)). Specifically the trace edge profiles considered are shown in Fig. 14, where the widths of the top ( $\left.w_{\text {top }}\right)$ and bottom

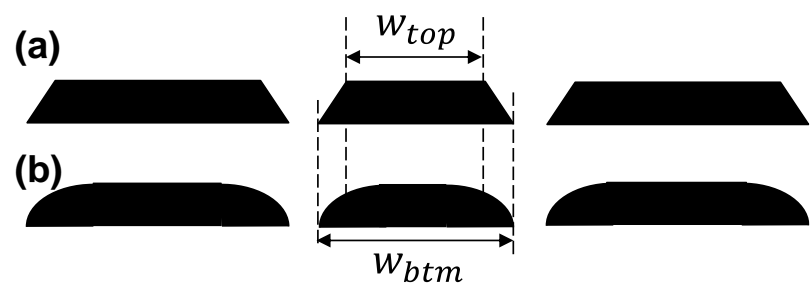

Fig. 14. Trace edge profile considered in simulation (a) Sharp trapezoidal edge; (b) Curved trapezoidal edge.

surfaces $\left(w_{b t m}\right)$ of the traces are modelled as identical, with the same conductor edge angles, with the only difference located in the trace edges. In modelling the trapezoidal corners as curved edges, a good match could be obtained between the simulation and measured results. This effect has been little investigated in literature [19] and the results indicate that the trace edge profile may affect CPW structures more significantly due to the confinement of the electric fields around the ground-signal-ground region. This phenomenon also agrees well with the observation made by Azucena et al. [20], in that the conductor losses are affected by the trace edge profiles of CPW structures since most of the current flows along the trace edges in those structures [20].

\section{VIII.CONCLUSION}

In this paper, the effect of the paste property on the DC conductivity and RF performance of screen printed traces was investigated. The important results of this work are summarized as follows:

1) The variation of the printed test structures in terms of the DC conductivity values was investigated for different regions of the substrate. Four point probe structures were positioned beside each CPW line, such that in-situ measurements of the DC conductivity values could be performed. From the results obtained, the average DC conductivity values were observed to vary across different regions of the RO3006 substrate, which may be attributed to the paste levelling after screen printing. This suggests that paste levelling has a significant influence on the DC electrical performance and necessitates a paste selection procedure prior to the actual printing of the test structures. The results also indicate the need for accurate DC conductivity values to be used for the simulation correlation of printed traces, since the values may vary across different regions of the substrate depending on the paste formulation and its interaction with the mesh and substrate during printing. 
2) The DC conductivity range of the traces on RO3006 was noted to be consistently higher compared to those on borosilicate glass, by a factor of 1.4-2 times. Further investigation revealed the presence of pin-holes and mesh marks on the traces printed on borosilicate glass, with thicknesses of around 6-7 $\mu \mathrm{m}$ which are below the recommended paste thickness. The results suggest that the trace quality and thickness are affected by the adhesion of the paste onto the particular substrate during the screen printing process. In this case a substrate with higher surface roughness (RO3006) possibly results in a stronger adhesion force, due to an increased contact area between the paste particles.

3) The measured RF performance for a $2 \mathrm{~mm}$ CPW line was validated with simulation results, where a good correlation was obtained. The results obtained are promising with respect to the goal of obtaining accurate $3 \mathrm{D}$ models to estimate the RF performance of printed traces.

\section{ACKNOWLEDGEMENT}

The authors are grateful to Rogers Corporation for the generous provision of the RO3006 laminates for this work. In addition, they are indebted to Computer Simulation Technology (CST) for the provision of the software license for this work, and to Dr Tracey Vincent (CST) for the technical support and helpful discussions rendered for this work. Lastly, the authors would like to acknowledge the 7th European Community Framework Programme for financial support through a Marie Curie International Research Staff Exchange Scheme (IRSES) Project entitled "Micro-Multi-Material Manufacture to Enable Multifunctional Miniaturised Devices (M6)" (Grant No. PIRSES-GA-2010-269113). A part of this work was conducted at the AIST Nano-Processing Facility and NIMS Nanofabrication Platform, supported by the "Nanotechnology Platform Program" of the Ministry of Education, Culture, Sports, Science and Technology (MEXT), Japan.

\section{REFERENCES}

[1] IEEE Standard for Local and metropolitan area networks-Part 15.4: Low-Rate Wireless Personal Area Networks (LR-WPANs), 2011.

[2] J-W. Kim, Y-C. Lee, J-M. Kim, W. Nah, H-S. Lee, H-C. Kwon, and SB. Jung, "Characterization of direct patterned Ag circuits for RF application", Microelectron. Eng., vol. 87, no. 3, pp. 379-82, Mar. 2010.

[3] K. Park, D. Seo, and J. Lee, "Conductivity of silver paste prepared from nanoparticles”, Colloids Surf. A, vol, 313-314, pp. 351-354, Feb. 2008.

[4] S. Jang, Y. Seo, J. Choi, T. Kim, J. Cho, S. Kim, and D. Kim, "Sintering of inkjet printed copper nanoparticles for flexible electronics", Scr. Mater. vol. 62, no. 5, pp. 258-261, Mar. 2010.

[5] K-S. Kim, W-R. Myung, and S-B. Jung, "Effects of sintering conditions on microstructure and characteristics of screen-printed Ag thin film", Electron. Mater. Lett., vol. 8, no. 3, pp. 309-314, Jun. 2012.

[6] C. Kim, M. Nogi, and K. Suganuma, "Electrical conductivity enhancement in inkjet-printed narrow lines through gradual heating", $J$. Micromech. Microeng. vol. 22, no. 3, 035016 (5 pp.), Feb. 2012.

[7] J. F. Salmeron, A.R. Torres, J. Banqueri, and M.A. Carvajal, "Design and characterization of ink-jet and screen printed HF RFID antennas," in Proc. Int. EURASIP Workshop on RFID Tech., Sept. 2012, pp. 119-23.

[8] J. F. Salmeron, F. Molina-Lopez, D. Briand, J.J. Ruan, A. Rivadeneyra, M.A. Carvajal, L. F. Capitan-Vallvey, N.F. Derooij, and A. J. Palma,
"Properties and printability of inkjet and screen-printed silver patterns for RFID antennas", J. Electron. Mater., vol. 43, no. 2, pp. 604-617, Feb. 2014.

[9] S Merilampi, T. Laine-Ma, and P. Ruuskanen, "The characterization of electrically conductive silver ink patterns on flexible substrates", Microelectron. Reliab., vol. 49, no. 7, pp. 782-790, Jul. 2009.

[10] I. Kazani, C. Hertleer, G. De Mey, A. Schwarz, G. Guxho, and L. Van Langenhove, "Electrical conductive textiles obtained by screen printing”, Fibres Text. East. Eur., vol. 20, no. 1(90), pp. 57-63, 2012.

[11] RO3000 Series Circuit Materials RO3003 ${ }^{\mathrm{TM}}$, RO3006 ${ }^{\mathrm{TM}}$ and RO3010 ${ }^{\mathrm{TM}}$ High Frequency Laminates. Rogers Corporation, 2011 [Online]. Available: https://www.rogerscorp.com. Accessed on April, 30, 2015.

[12] M. Aoki, K. Nakamura, T. Tachibana, I. Sumita, H. Hayashi, H. Asada, and Y. Ohshita, "30 $\mu \mathrm{m}$ Fine Line Printing for Solar Cells," in Proc. 39th IEEE Photovoltaic Specialists Conference (PVSC), Jun. 2013, pp. 2162-2166.

[13] J.W. Phair, M. Lundberg, and A. Kaiser, "Leveling and thixotropic characteristics of concentrated zirconia inks for screen-printing", Rheol. Acta, vol. 48, no. 2, pp. 121-33, Mar. 2009.

[14] D. Buzby and A. Dobie, "Fine Line Screen Printing of Thick Film Pastes on Silicon Solar Cells," in Proc. Int. Microelectron. Assem. Packag. Soc. (IMAPS), Nov. 2008, pp. 1-5.

[15] U. Itoh, M. Yoshida, H. Tokuhisa, N. Ushifusa, I. Sumita, T. Fukunishi, M. Aoki, Y. Ohshita, and H. Kamata, "Screen printed finger electrode with high aspect ratio by single printing for crystal Si solar cell using novel screen mask," in Proc. 38th IEEE Photovoltaic Specialists Conference (PVSC), Jun. 2011, pp. 002167-70.

[16] S. C. Joo, "Adhesion mechanisms of nano-particle silver to electronics packaging material," Ph.D. dissertation, The George W. Woodruff Sch. of Mech. Eng., Georgia Tech, 2009.

[17] Y. Y. Lim, Y. M. Goh and C. Liu, "Surface treatments for inkjet printing onto a PTFE-based substrate for high frequency applications", Ind. Eng. Chem. Res., vol. 52, no. 33, pp. 11564-74, Jul. 2013.

[18] BOROFLOAT® Borosilicate Float. Glass Abrisa Technologies, 2004, pp. 1-19.

[19] B. Curran, I. Ndip, S. Guttowski, and H. Reichl, "A methodology for combined modeling of skin, proximity, edge, and surface roughness effects", IEEE Trans. Microwave Theory Tech. vol. 58, no. 9, Sept. 2010.

[20] O. Azucena, J. Kubby, D. Scarbrough, and C. Goldsmith, "Inkjet printing of passive microwave circuitry," in Proc. Microwave Symp. Digest (MTT-S), 2008, pp. 1075-1078.

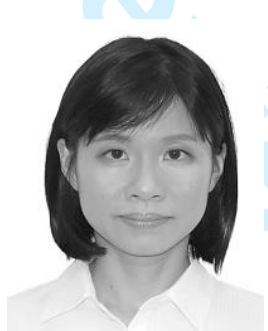

Ying Ying Lim received the B.Eng. degree and M.Eng degrees in electrical \& electronic engineering from Nanyang Technological University, Singapore, in 2003 and 2005. She received her Ph.D. degree in mechanical \& manufacturing engineering from Loughborough University, United Kingdom, in 2015.

From 2005 to 2011, she worked as a Research Engineer at the Institute of Microelectronics (IME), A*STAR, Singapore, where she designed, simulated and characterized high frequency embedded passives for packaging. She also has experience in the high frequency dielectric characterization of substrates and thin films. She is currently a post-doctoral researcher with the Nanoelectronics Research Institute (NeRI) at AIST.

Dr. Lim was the recipient of the Outstanding Student Paper Award at the Electronics Packaging Technology Conference (EPTC 2005), and the Best Interactive Poster Award at Third International Conference in Advanced Manufacturing for Multifunctional Miniaturised Devices (ICAM3D-2014). Her current research interest is in the area of wearable electronics. 


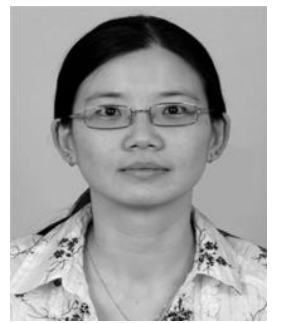

Yee Mey Goh has BEng (Hons) in Mechanical Engineering from University Tenaga Nasional, Malaysia and a PhD from University of Bristol, UK. She joined Loughborough University in 2010 and is currently a Senior Lecturer in Wolfson School of Mechanical, Electrical and Manufacturing Engineering. Her inter-disciplinary research focusses on the development of data and knowledge-driven models and methods to support through-life engineering of product-service systems and intelligent automation. Her expertise includes uncertainty analysis, machine learning, wearable technologies and decision support for broad application in engineering design, energy systems and manufacturing within aerospace, defence, energy and automotive sectors. She is a member of the Design Society and the scientific committee for DESIGN, EP\&DE, ICED and ASME IDETC international conferences. She is a committee member of the consortium of UK University Manufacturing and Engineering (COMEH).

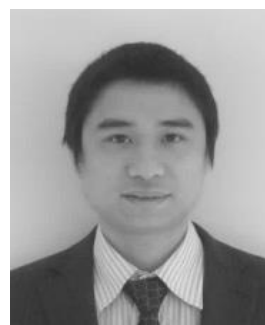

Bui Thanh Tung received the B.S. degree in electrical engineering from Vietnam National University, Hanoi (VNUH) in 2004, and the M.E. and D.Eng. degrees in Science and Engineering from Ritsumeikan University, Shiga, Japan, in 2008 and 2011, respectively. From 2011 to 2015 he was a post-doctoral researcher with the 3D Integration System Group, Nanoelectronics Research Institute (NeRI), National Institute of Advanced Industrial Science and Technology (AIST), Tsukuba, Japan. Currently, he is an assistant professor at the Faculty of Electronics and Telecommunication (FET), University of Engineering and Technology (UET), Vietnam National University, Hanoi (VNUH). His current interests include MEMS based sensors, actuators and applications.

Manabu Yoshida received the B.A in materials science and PhD (Tech). from Chiba University in 1994 and 1999. From 1999 to 2001 he was a CREST researcher. From 2001 to 2009 he worked as a researcher of the Organic Semiconductor Device Group in the National Institute of Advanced Industrial Science and Technology (AIST). From 2009-2011, he was a senior researcher in the organic semiconductor device group in AIST. From 2011-2012, he was a senior researcher in the printed electronics device team (FLEC) in AIST. From 2012-2013, he was director of the Electronics, Materials Technology and Nanotechnology Department of NEDO, Japan. He is currently a team leader of the printed electronics device team (FLEC) in AIST. His expertise includes printed electronics, organic electronics and flexible and stretchable electronics.

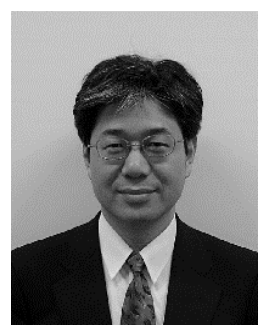

Masahiro Aoyagi (M'94-SM'10) received the B.E. and D.E. degrees in electronic engineering from Nagoya Institute of Technology, Japan, in 1982 and 1991, respectively. He joined Electrotechnical Laboratory, Tsukuba, Japan, in 1982, where he has been engaged in the research and development of $\mathrm{Nb}$, $\mathrm{NbN}$ superconducting devices and Josephson integrated circuits. He worked in the special section Josephson computer technology from 1982 to 1994 . He worked as a guest researcher in National Physical Laboratory, Teddington, UK, from 1994 to 1995 . He was a group leader of High Density Interconnection Group, Nanoelectronics Research Institute (NeRI), National Institute of Advanced Industrial Science and Technology (AIST) from 2000 to 2010. He worked as a group leader in the national R\&D projects of High Density Electronic System Integration from 1999 to 2004 and Functionally Innovative 3D-Integrated Circuit Technology from 2008 to 2012. He was the Deputy Director of NeRI, AIST from 2012 to 2014. He is currently the Director of Collaboration Promotion Unit, TIA Central Office, AIST. His present research field is high-performance high-density 3D system integration technology.

Dr. Aoyagi was awarded the Tsukuba prize of 1991 for the development of Josephson prototype computer ETL-JC1. He has authored or co-authored 340 technical papers and has 150 patents. He was the chair of IEEE Components, Packaging and Manufacturing Technology (CPMT) Japan Chapter from 2009 to 2010. He is a member of Board of Governors (BoG) for IEEE CPMT from 2012. 